\title{
ON HYPERELLIPTIC SCHOTTKY GROUPS
}

\author{
LINDA KEEN
}

\section{Section 1: Introduction}

A compact Riemann surface of genus $g$ is hyperelliptic if it is a two sheeted covering of the Riemann sphere branched at $2(g+1)$ points; that is, if it satisfies a polynomial equation of the form:

$$
w^{2}=\left(z-a_{0}\right)\left(z-a_{1}\right)\left(z-a_{2}\right) \ldots\left(z-a_{2 g+1}\right),
$$

where $a_{k}, k=0, \ldots, 2 g+1$ are distinct points on the Riemann sphere. There are various characterizations of hyperelliptic surfaces in the literature but none of them involve uniformization theory. In uniformization theory we begin with a surface $S$, a covering space $\tilde{S}$ and the corresponding group of cover transformations $G$. If we can realize $\tilde{S}$ as a plane domain $\Omega$, and $G$ as a group of linear fractional transformations $\Gamma$, such that the projection map $\pi: \Omega \rightarrow \Omega / \Gamma=S$ is holomorphic, then $\Gamma$ is said to uniformize $S$. Schottky groups are realizations of the smallest covering groups which correspond to planar covering surfaces. In this paper we will discuss those Schottky groups which uniformize hyperelliptic surfaces.

We begin with a discussion of some geometric properties of linear fractional transformations and their interpretation when the linear fractional transformations are represented as elements of $S L(2, \boldsymbol{C})$. In Section 3 we discuss Schottky groups in some detail and describe a set of moduli for them. In Section 4 we consider surfaces of genus two, all of which are hyperelliptic, and determine various properties of the corresponding Schottky groups. Section 5 contains the main theorem, which gives a description of the moduli space of those Schottky groups which uniformize hyperelliptic groups and which reflect their hyperellipticity.

\section{Section 2: Linear fractional transformations}

In this section we state some facts about the relationships between the fixed points of linear fractional transformations, the geometry of circles in the plane, hyperbolic three space and Lie brackets of matrices in $S L(2, \boldsymbol{C})$. In particular, we determine a generalization of the concept of the intersection point of the axes of a pair of hyperbolic elements of a Fuchsian group. 
Definition 1. Let $(p, q)$ and $(u, v)$ be two pairs of points in the complex plane. They are called harmonic pairs if the cross ratio $(p, q, u, v)$ has the value -1 .

Geometrically, this means that if we form the hyperbolic pencil $\tilde{H}$ of circles through $(p, q)$ and the elliptic pencil $\widetilde{E}$ of circles orthogonal to those of $\widetilde{H}$, then $(u, v)$ are the intersection points of a circle in $\tilde{H}$ with one in $\tilde{E}$.

Proposition 2.1. If we are given two distinct pairs of points $(p, q)$ and $(r,(s$ there is a unique pair of points $(u, v)$ which is harmonic with respect to both pairs.

Proof. First, assume the points in each pair are also distinct. There is a unique linear fractional transformation $T$ which sends $p$ into $q, q$ into $p$, and $r$ into $s$. A transformation which interchanges a pair of points must be an involution, so $T(s)=r$. Its fixed point pair $(u, v)$, is uniquely determined and is harmonic with respect to both pairs $(p, q)$ and $(r, s)$.

If $p=q$ or $r=s$ then this point will be one of the points $(u, v)$. If $p=q$ and $r=s$ then $p=u$ and $r=v$ ([Fenchel, 2]).

Definition 1 a. Let $A$ and $B$ be a pair of linear fractional transformations and let $\left(p_{A}, q_{A}\right),\left(p_{B}, q_{B}\right)$ be their respective fixed point pairs. The pair of points $(u, v)$ harmonic with respect to both pairs $\left(p_{A}, q_{A}\right)$ and $\left(p_{B}, q_{B}\right)$ will also be called the pair harmonic with respect to $(A, B)$.

When $p_{A}, q_{A}, p_{B}$ and $q_{B}$ are distinct and lie on a circle $C$, the group generated by $A$ and $B$ is Fuchsian; $(u, v)$ are the intersection points inside and outside the circle $C$ of the axes of $A$ and $B$ - the axis of $A$ (resp. $B$ ) is the circle through $p_{A}$ and $q_{A}$ (resp. $p_{B}$ and $q_{B}$ ) which is orthogonal to the circle $C$; inside (or outside) it is the unique geodesic joining the fixed points in the hyperbolic metric.

If we consider our transformations $A, B$ not only as mappings of the complex plane but also as isometries of hyperbolic three space $H^{3}$, we can give another interpretation of $(u, v)$. There is a unique geodesic $h_{A}$ in $H^{3}$ which joins $p_{A}$ and $q_{A}$ which is invariant under the transformation $A$. (Here $h_{A}$ is the circle through $p_{A}$ and $q_{A}$ orthogonal to the complex plane.) $h_{A}$ is called the $H^{3}$-axis of $A$. Let $h_{B}$ be the $H^{3}$-axis of $B$. There is a unique perpendicular to $h_{A}$ and $h_{B}$ in $H^{3}$. It has endpoints $(u, v)$ in the complex plane.

The linear transformations can be represented as elements of $S L(2, C) / \pm I$. Given $A$ and $B$ we can form the Lie bracket $\tilde{E}=A B-B A$. Let $\delta=\operatorname{det} \widetilde{E}$ and $E=\delta^{-1 / 2} \tilde{E}$. Then $\delta \neq 0$ and $E$ belongs to $S L(2, C)$ provided $\left(p_{A}, q_{A}\right)$ and $\left(p_{B}, q_{B}\right)$ are disjoint pairs. The fixed points of $E$ are $(u, v)$ and $E^{2}=-I$ ( $I$ is the identity in $S L(2, \boldsymbol{C}))$.

The proof that $(u, v)$ are the endpoints of the hyperbolic perpendicular is not difficult but is not appropriate here since we will not need the techniques for anything else in this paper. The proof that $(u, v)$ are the fixed points of the Lie bracket is a straightforward computation which uses the fact that there is only one elliptic transformation of order two which has $(u, v)$ as fixed points (see [Jørgensen, 4]). 
Proposition 2.2. Let $A$ and $B$ be linear fractional transformations with no common fixed point. Let $(u, v)$ be harmonic with respect to $(A, B)$. Then for any pair $m, n$ of non-zero integers:

i) $(u, v)$ is harmonic with respect to $(B, A)$ and $\left(A^{n}, B^{m}\right)$,

ii) $(u, v)$ is harmonic with respect to $(A B, B A)$,

iii) $(u, v)$ is harmonic with respect to $\left(A, A^{n} B^{m} A^{n}\right)$,

iv) if $(u, v)$ is also harmonic with respect to $(A, C)$, then it is harmonic with respect to $(B, C)$.

Proof. (i) follows from Definition 1 a since $A$ and $B$ appear symmetrically and it is only their fixed points which are relevant. We prove (ii) and leave (iii) and (iv) to the reader, since the proofs are similar. First we recall that two linear fractional transformations commute if, and only if, they have common fixed points. For $E=A B-B A$, with equality taken as equality of linear transformations, we have:

$$
\begin{gathered}
E^{-1}=B^{-1} A^{-1}-A^{-1} B^{-1} . \\
=(A B-B A)\left(A B^{-1}-B^{-1} A\right) A^{-1} .
\end{gathered}
$$

By part (i), $A B^{-1}-B^{-1} A=E$ so $E A E=A^{-1}$. Similarly $E B E=B^{-1}$. Set $F=A B B A-$ $B A A B$ and look at $E F E=E A B^{2} A E-E B A^{2} B E=A^{-1} B^{-2} A^{-1}-B^{-1} A^{-2} B^{-1}=$ $F^{-1}=F$. Since $E$ and $F$ commute they have the same fixed points. They are both elliptic transformations of order two hence they are equal.

\section{Section 3: Schottky groups}

Let $C_{1}, C_{1}^{\prime}, \ldots, C_{g}, C_{g}^{\prime}, g \geqq 2$, be $2 g$ Jordan curves on the Riemann sphere $\hat{C}$ such that the curves are mutually disjoint and bound a $2 g$-connected domain $D$. Call $D$ the common exterior of all the curves, and suppose that for each $k, k=1, \ldots, g$, there exists a linear fractional transformation $A_{k}$ with the properties:

i) $A_{k}\left(C_{k}\right)=C_{k}^{\prime}$;

ii) $A_{k}$ maps the exterior of $C_{k}$ onto the interior of $C_{k}^{\prime}$.

The transformations $A_{k}$ must be loxodromic, the group $\Gamma$ they generate is necessarily discontinuous and $D$ is a fundamental domain for $\Gamma$. $\Gamma$ is called a Schottky group of genus $g$. Let $\Omega=\cup_{A \in \Gamma} A(D) ; \Omega$ is the set of discontinuity, or the regular set of $\Gamma$. The limit set, $\Lambda=\hat{C}-\Omega$, is closed, perfect and totally disconnected. We summarize the main results we need about Schottky groups below.

Theorem 3.1 [Maskit, 9]. A group of linear fractional transformations with a non-empty domain of discontinuity (Kleinian group) is a Schottky group if, and only if, it is finitely generated, free and purely loxodromic. 
Theorem 3.2 [Chuckrow, 1]. If $\Gamma$ is a Schottky group, then corresponding to any set of free generators, there is a fundamental domain $D$, as above, whose boundary curves are identified by the given generators.

Theorem 3.3 [Maskit, 10]. If $\Gamma$ and $\Gamma^{\prime}$ are two Schottky groups with regular sets $\Omega$ and $\Omega^{\prime}$, and limit sets $\Lambda$ and $\Lambda^{\prime}$ respectively, and if $\varphi$ is a type-preserving isomorphism, $\varphi: \Gamma \rightarrow \Gamma^{\prime}$, then there is a quasiconformal mapping $w: \Omega \rightarrow \Omega^{\prime}$, such that $\Gamma^{\prime}=w \circ \Gamma \circ w^{-1}$, and, moreover, $w$ can be extended as a quasiconformal homeomorphism to $\Lambda, w: \Lambda \rightarrow \Lambda^{\prime}$.

The image $S$, of the natural projection mapping $\pi, \pi: \Omega \rightarrow \Omega / \Gamma=S$, is a compact Riemann surface of genus $g$. The boundary curves, $C_{k}, C_{k}^{\prime}, k=1, \ldots, g$, project onto a set of $g$ simple, mutually disjoint, homologically independent loops on the surface $S$. Conversely, if we begin with a surface $S$, on which such a set of loops is given, and form the highest regular covering $\tilde{S}$ for $S$ such that these loops lift to loops, (the defining subgroup for this covering is the smallest normal subgroup of $\Pi_{1}(S)$ containing the loops) we can use Maskit's planarity theorem, [8], and uniformization theory to obtain a Schottky group which uniformizes $S$. The Schottky group is uniquely determined by $S$ and the set of loops - up to conjugation by an arbitrary linear fractional transformation. Although the group is uniquely determined, the generators are not. If we choose a base point $b$ for the fundamental group, and a set of paths from $b$ to the loops, however, we do define a set of generators, or marking, for the group. Different sets of loops on the surface can lead to distinct Schottky groups.

Since a linear fractional transformation is uniquely determined by its trace and its fixed points, it is not hard to prove:

Theorem 3.4. Let $\mathscr{S}$ be the space of marked Schottky groups,

$$
\mathscr{S}=\left\{\Gamma=\left\langle A_{1}, A_{2}, \ldots, A_{g}\right\rangle\right\},
$$

let $x_{k}=$ trace $A_{k}$, and let the fixed points of $A_{k}$ be $r_{k}$ and $s_{k}, k=1, \ldots, g$. Assume $r_{1}=1, s_{1}=-1, r_{2}=-s_{2}$, then a set of moduli for $\mathscr{S}$ is the set of $3_{g}-3$ complex numbers, $x_{1}, \ldots, x_{g}, s_{2}, r_{3}, s_{3}, \ldots, r_{g}, s_{g}$.

\section{Section 4}

In [5] and [6] we considered pairs of punctured tori uniformized by Fuchsian and quasi-Fuchsian groups respectively. These groups are on the boundary of the Schottky space of surfaces of genus two, and in this section we will show that some of the properties we found for boundary groups extend into the interior.

First of all, every surface $S$ of genus two is hyperelliptic and so admits an involution $h$. Set $S_{0}=S / h ; S_{0}$ is a surface of genus zero with six ramification points $\left(p_{0}, \ldots, p_{5}\right)$, each of order two. 
Second, any Schottky group $\Gamma$ which represents $S$ is a free group on two generators. Let $\Gamma=\langle A, B\rangle$, let $\Omega=\Omega(\Gamma)$ be its regular set, and $\pi_{\Gamma}=\pi: \Omega \rightarrow S$ be the natural projection. Also let $E_{0}=A B-B A$. The fixed points $\left(p_{0}, q_{0}\right)$ of $E_{0}$ are harmonic with respect to the transformations $A$ and $B$.

Theorem 4.1. i) We can form an extension $G$ of $\Gamma, G=\left\langle E_{0}, \Gamma\right\rangle$ such that $\Gamma$ has index two in $G$.

ii) $E_{0}$ commutes with $h$; i.e., $h \circ \pi=\pi \circ E_{0}$.

iii) $E_{1}=E_{0} A$ and $E_{2}=E_{0} B$ are also elliptic of order two; and if the fixed points of $E_{i}$ are $\left(p_{i}, q_{i}\right), i=0,1,2$, then the fixed points of $h$, are precisely $\pi\left(p_{0}\right), \pi\left(q_{0}\right), \pi\left(p_{1}\right)$, $\pi\left(q_{1}\right), \pi\left(p_{2}\right)$ and $\pi\left(q_{2}\right)$.

Proof. Assume $S$ has no other conformal self mappings except $h$ - the generic case. Normalize so that

$$
E_{0}=\left(\begin{array}{rr}
i & 0 \\
0 & -i
\end{array}\right) \quad \text { and } \quad A=\frac{1}{2}\left(\begin{array}{lr}
x & -X \\
-X & x
\end{array}\right) .
$$

It follows that $B$ must have the form

$$
B=\frac{1}{2}\left(\begin{array}{lr}
y & -Y r \\
-Y / r & y
\end{array}\right)
$$

and that the fixed points of $E_{0}$ are $(0, \infty)$, those of $A$ are $(+1,-1)$ and those of $B$ are $(+r,-r)$, where $X^{2}=x^{2}-4, Y^{2}=y^{2}-4$. The proof of Proposition 2.2 implies $E_{0} A E_{0}=A^{-1}, E_{0} B E_{0}=B^{-1}$ hence $E_{0} \Gamma E_{0}=\Gamma, E_{0}(\Omega)=\Omega$ and $G=\left\langle E_{0}, \Gamma\right\rangle$ is a $Z_{2}$ extension of $\Gamma$, as asserted in i).

The map $E_{0}: \Omega \rightarrow \Omega$ induces a conformal mapping of $S$ onto itself, of order 2. Since the only conformal selfmapping $S$ admits is $h$, we have proved (ii).

To prove (iii), write down $E_{1}=E_{0} A$ and $E_{2}=E_{0} B$. They are clearly elliptic and their fixed points, together with those of $E_{0}$ project to fixed points of $h$ on $S$. To see these are distinct, note that $A$ and $B$ are distinct coset representatives of $G / \Gamma$ and that therefore $E_{0}, E_{1}$ and $E_{2}$ are a set of generators for $G$.

We give another proof of (ii) which, while it is not as simple as the one above, sheds more light on the situation in higher genus. Let $D$ be a fundamental domain for $\Gamma$ with boundary curves $\gamma_{1}, A_{1}\left(\gamma_{1}\right), \gamma_{2}$, and $A_{2}\left(\gamma_{2}\right)$. Each pair, $\left(\gamma_{i}, A_{i}\left(\gamma_{i}\right)\right)$ projects onto a simple closed curve $\bar{\gamma}_{i}$ on $S, i=1,2$. Let $\Pi_{1}(S, b)$ be the fundamental group of $S$ with base point $b$, and choose a path $\sigma_{i}$ from $b$ to $\bar{\gamma}_{i}$. The curves $\delta_{i}=\sigma_{i} \bar{\gamma}_{i} \sigma_{i}^{-1}$ determine the Schottky group $\Gamma$ with its marking $\langle A, B\rangle$, up to normalization. Similarly, the curves $\bar{\delta}_{i}=h\left(\delta_{i}\right)$ determine another Schottky group $\bar{\Gamma}$ with a marking $\langle\bar{A}, \bar{B}\rangle$ which can also be normalized. Normalize both these groups as in the hypothesis. Using the isomorphism $\varphi: \Gamma \rightarrow \Gamma$ given by $\varphi(A)=\bar{A}, \varphi(B)=\bar{B}$, we can lift $h$ to a mapping $H: \Omega(\Gamma) \rightarrow \bar{\Omega}(\bar{\Gamma})$ so that $\pi_{\Gamma} \circ H=h \circ \pi_{\Gamma}$.

The groups $\Gamma$ and $\bar{\Gamma}$ are both Schottky, so $H$ can be extended to the limit set $\Lambda(\Gamma)$ (Theorem 3.3) and, since $h$ is conformal, $H$ is a linear fractional transforma- 
tion. Since $h$ has order two, $H$ is elliptic of order 2. We have $\bar{\Gamma}=H \circ \Gamma \circ H^{-1}$; if we form $\bar{E}_{0}=\bar{A} \bar{B}-\bar{B} \bar{A}$ and $\bar{G}=\left\langle\bar{\Gamma}, \bar{E}_{0}\right\rangle$ we also have $\bar{G}=H \circ G \circ H^{-1}$ and in particular $\bar{E}_{0}=H \circ E_{0} \circ H^{-1}$. However, we have normalized both groups so that $E_{0}=\bar{E}_{0} . H$ commutes with $E_{0}$ and therefore has the same fixed points. Since it is also elliptic of order two $H=E_{0}$. Consequently, $\bar{\Gamma}=\Gamma$ and $H=E_{0}$ is the lift of $h$.

Corollary 4.1. Let $\Gamma=\langle C, D\rangle$ be any marking for the group $\Gamma$. Then $E=C^{n} D^{m}-D^{m} C^{n}$ belongs to $G$, for any pair of integers, $m$ and $n$.

Proof. Apply elementary Nielsen transformations to the pair of generators $\langle A, B\rangle$ and use Proposition 2.2 in Section 2.

Theorem 4.1 and Corollary 4.1 imply

Corollary 4.2. Let $S$ be a surface of genus two and let $\Gamma$ be any Schottky group representing $S$. The fixed points of the hyperelliptic involution can always be determined as follows: let $\langle A, B\rangle$ be any set of generators for $\Gamma$; then the fixed points of the Lie brackets, $\{A, B\},\left\{A, A^{-1} B\right\}$ and $\left\{A, B A^{-1}\right\}$, project to the fixed points of $h$.

The presentation $G=\left\langle E_{0}, E_{1}, E_{2}\right\rangle$ of the group $G$ reflects better than the presentation $G=\left\langle E_{0}, \Gamma\right\rangle$, the fact that the underlying surface, $S_{0}=\Omega(G) / G$, is a sphere with six ramification points of order two. Since these six points are the images of the fixed points of the elliptic transformations, they are paired by the transformations. This pairing depends on the group $G$, and hence $\Gamma$, but it is independent of the marking for $\Gamma$. If we begin with the surface $S_{0}=\Omega(G) / G$, pair the ramification points, and then choose three loops in $\Pi_{1}\left(S_{0}\right)$ each of which separates one pair of points from the other two pairs, we determine a group $\widetilde{G}=\left\langle\widetilde{E}_{0}, \widetilde{E}_{1}, \widetilde{E}_{2}\right\rangle$. In general, the groups $G$ and $\widetilde{G}$ are not the same. Indeed, as Figure 1 indicates, the different choices of loops do lead to different groups. We will not pursue here the question of which choices do lead to the same - but differently marked groups.

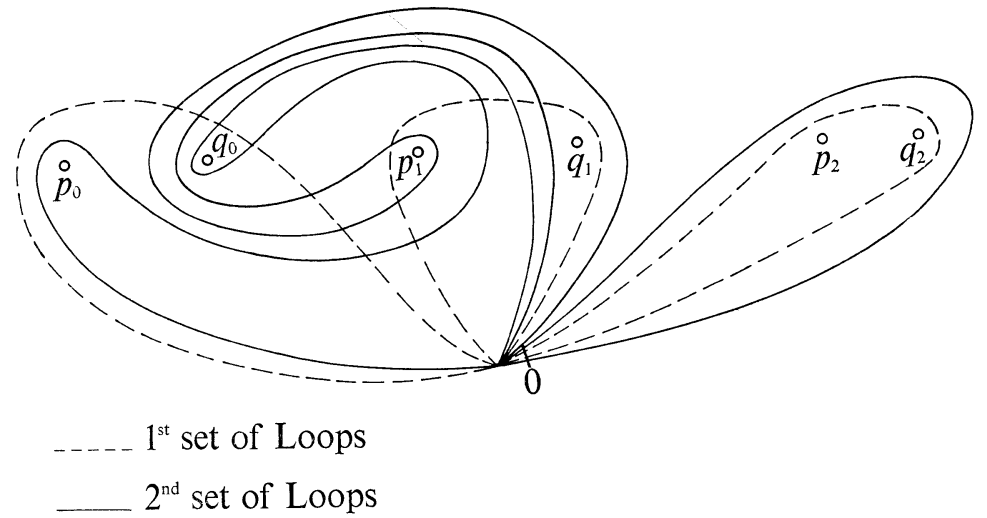

Figure 1 
In Figure 2 we indicate a fundamental domain $\Delta$ for $G$; such always exists ([1]). It is bounded by three disjoint simple closed curves $C_{0}, C_{1}, C_{2}$, such that $C_{i}$ contains $p_{i}$ and $q_{i}$ and is invariant under the elliptic transformation $E_{i}, i=0,1,2$. A fundamental $D$ domain for $\Gamma$ is $D=\Delta \cup E_{0}(\Delta)$.
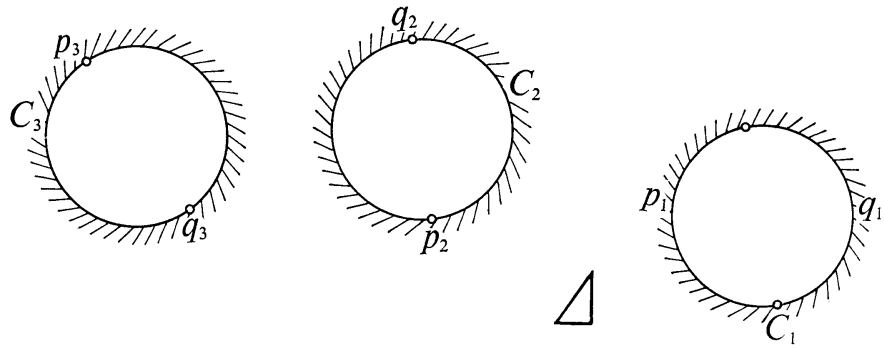

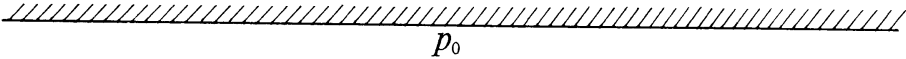
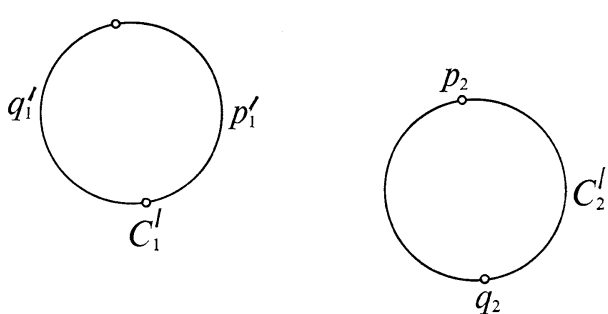

$E_{0}(\triangle)$

Figure 2

\section{Section 5}

Suppose now that $S$ is a hyperelliptic Riemann surface of genus $g, g>2$. Using the previous section as a guide, we want to determine which Schottky uniformizations of $S$ contain the information that $S$ is hyperelliptic.

To this end, we first construct the genus $g$ analogue of the group $G$ of the previous section, with its fundamental domain $\Delta$. Let $\left(p_{0}, q_{0}\right),\left(p_{1}, q_{1}\right), \ldots,\left(p_{g}, q_{g}\right)$ be $g+1$ pairs of points which lie in a half plane. Suppose that $p_{0}=0, q_{0}=\infty$. The elliptic transformation $E_{k}$ of order two which has $p_{k}, q_{k}$ as fixed points has the form

$$
E_{k}=\frac{i}{q_{k}-p_{k}}\left(\begin{array}{cc}
p_{k}+q_{k} & -2 p_{k} q_{k} \\
2 & -\left(p_{k}+q_{k}\right)
\end{array}\right) \quad k=1, \ldots, g, E_{0}=\left(\begin{array}{rr}
i & 0 \\
0 & -i
\end{array}\right) \text {. }
$$

If the points are sufficiently far apart we can find curves $C_{k}$ passing through $\left(p_{k}, q_{k}\right)$ and containing $\left(p_{j}, q_{j}\right)$ in their exterior, $j \neq k$, such that $E_{k}\left(C_{k}\right)=C_{k}$. Although $G=\left\langle E_{0}, \ldots, E_{g}\right\rangle$ is not a Schottky group, the common exterior of the curves $C_{k}$, $k=0, \ldots, g$, is a fundamental domain $\Delta$ for it. Such a group is called a Schottkytype group. The group $\Gamma$ generated by $\left\langle A_{1}, \ldots, A_{g}\right\rangle$, where $A_{k}=E_{0} E_{k}, k=1, \ldots, g$, 
is a Schottky group with fundamental domain $D=\Delta \cup E_{0}(\Delta)$. The elements $A_{k}$ have the form

$$
A_{k}=\frac{1}{2}\left(\begin{array}{lc}
x_{k} & -X_{k} t_{k} \\
-X_{k} / t_{k} & x_{k}
\end{array}\right) \text { where } \quad x_{k}=\frac{2\left(p_{k}+q_{k}\right)}{p_{k}-q_{k}},
$$

$X_{k}^{2}=x_{k}^{2}-4$ and $t_{k}=\sqrt{p_{k} q_{k}}$. The fixed points of $A_{k}$ are $t_{k}$ and $-t_{k}$. We see that $(0, \infty)$ is harmonic with respect to $\left(A_{j}, A_{k}\right), j \neq k, 1 \leqq j, k \leqq g$.

Definition. Let $\Gamma=\left\langle A_{1}, \ldots, A_{g}\right\rangle$ be a Schottky group and let $(p, q)$ be a pair of points such that $(p, q)$ is harmonic with respect to any pair of generators $\left(A_{j}, A_{k}\right)$. Then we say $\left\langle A_{1}, \ldots, A_{g}\right\rangle$ is a mutually harmonic set of generators for $\Gamma$.

Lemma 1. If $\Gamma$ is a Schottky group which admits a mutually harmonic set of generators it represents a hyperelliptic surface.

Proof. Given $\Gamma=\left\langle A_{1}, \ldots, A_{g}\right\rangle$, set $E_{0}=A_{1} A_{2}-A_{2} A_{1}$. If we assume $(p, q)=$ $(0, \infty)$, the condition of being mutually harmonic becomes the condition that the fixed points $t_{k}, s_{k}$ of $A_{k}$ satisfy $s_{k}=-t_{k}$ for all $k=1, \ldots, g$. Moreover, $E_{0}=A_{j} A_{k}-$ $A_{k} A_{j}$ for any distinct pair of generators $A_{j}$ and $A_{k}$. The elements $E_{k}=E_{0} A_{k}$ are also elliptic of order two, and we can look at the extended group $G=\left\langle\Gamma, E_{0}\right\rangle$. Since $E_{0} A_{k} E_{0}=A_{k}^{-1}, \Gamma$ has index 2 in $G$, the projection $\Omega \rightarrow \Omega / G$ is well defined and the quotient can be identified as a sphere ramified at $2 g+2$ points. Moreover since $G$ is normal in $\Gamma$ we obtain a covering $S=\Omega / \Gamma \rightarrow \Omega / G$ which is two to one except at the ramification points. The "sheet interchange" lifts to the map $E_{0}: \Omega \rightarrow \Omega$.

Lemma 2. If $S$ is hyperelliptic it can be uniformized by a Schottky group which admits a mutually harmonic set of generators.

Proof. Let $h$ be the hyperelliptic involution of $S$. On $S / h=S_{0}$ determine a pairing of the ramification points. We can find a set of $g+1$ loops each of which separates one pair of ramification points from all the other pairs. These loops determine a Schottky type group $G, G=\left\langle E_{0}, \ldots, E_{g}\right\rangle$, uniformizing $S_{0}$. The subgroup $\Gamma=\left\langle A_{1}, \ldots, A_{g}\right\rangle$ of $G$, where $A_{k}=E_{0} E_{k}, k=1, \ldots, g$, has index 2 in $G$, has a mutually harmonic set of generators and uniformizes $S$. Clearly, the elements $E_{k}$, which conjugate $\Gamma$, project to the hyperelliptic involution $h$.

Lemma 3. If $\Gamma$ is a Schottky group representing a hyperelliptic surface $S$ and if $\Gamma$ is invariant under the hyperelliptic involution $h$, then $\Gamma$ admits a mutually harmonic set of generators.

Proof. Let $\Gamma$ be given with presentation $\Gamma=\left\langle B_{1}, \ldots, B_{g}\right\rangle$ and let $D$ be a fundamental domain for $\Gamma$ corresponding to this set of generators. Project the boundary curves of $D$ to loops $\left(\gamma_{1}, \ldots, \gamma_{g}\right)$ on $S$. Choose a base point $b$ on $S$ and paths $\sigma_{1}, \ldots, \sigma_{g}$ from $b$ to $\gamma_{k}$ so that $\bar{\gamma}_{k}=\sigma_{k} \gamma_{k} \sigma_{k}^{-1}, k=1, \ldots, g$, form a set of elements of $\Pi_{1}(S, b)$ which give rise to the marked group $\Gamma=\left\langle B_{1}, \ldots, B_{g}\right\rangle$. The elements $h\left(\bar{\gamma}_{k}\right) \in \Pi_{1}(S, b)$ give rise to the marked group $\bar{\Gamma}=\left\langle\bar{B}_{1}, \ldots, \bar{B}_{g}\right\rangle$. The assumption that $\Gamma$ is invariant 
under $h$ says that $\bar{\Gamma}=\Gamma$. We have an automorphism $\varphi: \Gamma \rightarrow \bar{\Gamma}$ given by $\varphi\left(B_{k}\right)=\bar{B}_{k}$, $k=1, \ldots, g$. Lift $h$ to $\Omega, H: \Omega \rightarrow \Omega$ using this automorphism. As in the genus two case, $H$ can be extended to the limit set and must be a linear fractional transformation which is elliptic of order two. Rename $H, E_{0}$; renormalize so that $E_{0}$ has fixed points $(0, \infty)$ and so that they belong to $D$.

$\pi(0)$ and $\pi(\infty)$ are two of the fixed points of $h$. Let $\tilde{p}_{1}$ be another, and $p_{1}$ a lift of $\tilde{p}_{1}$ in $D$. Since $\pi \circ E_{0}\left(p_{1}\right)=h\left(p_{1}\right)=p_{1}$, and since $E_{0} \Gamma E_{0}=\Gamma$ by assumption, there is an element $A_{1} \in \Gamma$ such that $A_{1}\left(p_{1}\right)=E_{0}\left(p_{1}\right) . E_{1}=E_{0} A_{1}$ is elliptic of order two. To see this, set $\bar{A}_{1}=E_{0} A_{1} E_{0}$. Then, $E_{1}=E_{0} A_{1}=\bar{A}_{1} E_{0}$ so $E_{1}^{2}=\bar{A}_{1} A_{1} \in \Gamma$. Since $p_{1}$ is a fixed point of $E_{1}^{2}$ and $p_{1} \in \Omega, E_{1}^{2}$ must be the identity. Let $q_{1}$ be the other fixed point of $E_{1}$. Continue in this manner to define elements $E_{1}, \ldots, E_{g}$ where each $E_{k}, k=2, \ldots, g$ is elliptic of order two and has fixed points $p_{k}, q_{k}$. The projections of these points constitute the full set of $2 g+2$ fixed points of the hyperelliptic involution. The elements $A_{1}, \ldots, A_{g}, A_{k}=E_{0} E_{k}, k=1, \ldots, g$ form a mutually harmonic set. We claim they generate $\Gamma$.

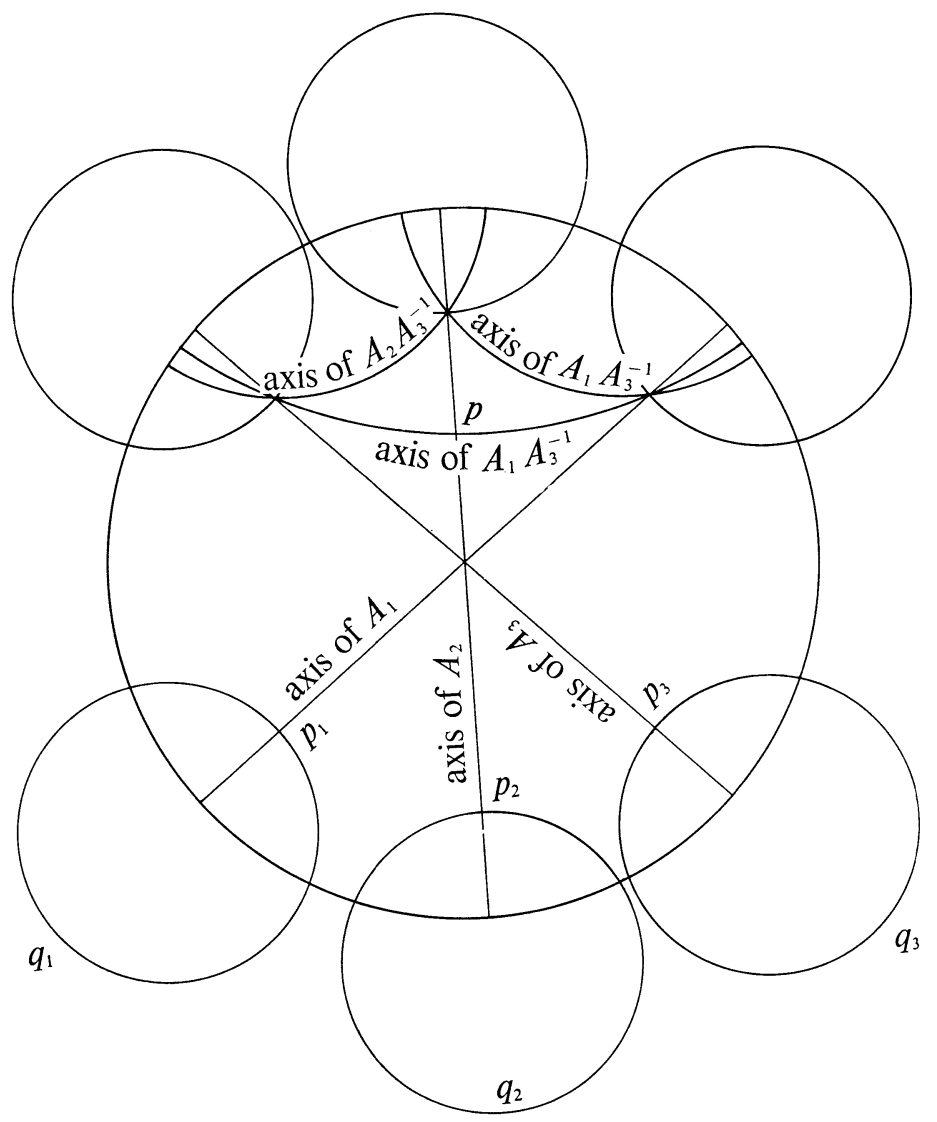

Figure 3 
Let $G=\left\langle\Gamma, E_{0}\right\rangle . \Gamma$ is a subgroup of index two in $G$ since $\Gamma=E_{0} \Gamma E_{0}$. We can also write $G=\left\langle\Gamma, E_{k}\right\rangle, k=1, \ldots, g$; each $E_{k}$ preserves the set of fixed points of elliptic elements of $G$ so that $G=\left\langle E_{0}, \ldots, E_{g}\right\rangle$. Now since the $A_{k}$ also generate a subgroup of index two in $G$, that subgroup must be $\Gamma$. Taken together these lemmas imply:

Theorem 5.1. The sublocus of the moduli space $\mathscr{S}$ of Theorem 3.4, which is defined by the $g-2$ equations $s_{k}=-t_{k}, k=2, \ldots, g$, consists precisely of those Schottky groups which represent hyperelliptic surfaces and which are invariant under the hyperelliptic involution.

Remark. Suppose $\Gamma$ is a Schottky group with a mutually harmonic set of generators $\left\langle A_{1}, \ldots, A_{g}\right\rangle, g>2$. It is no longer the case, as it was when $g=2$, that the extended group $G=\left\langle\Gamma, E_{0}\right\rangle$ contains the Lie bracket of any pair of elements, which constitute part of a set of generators for $\Gamma$. To see this, consider for example, Figure 3 where we have taken $\Gamma$ to be a Fuchsian group of the second kind and $S$ to be a surface of genus three. We can find harmonic points easily since we need only draw axes. The point $p$ indicated there is the fixed point of the Lie bracket $\left\{A_{3}^{-1} A_{1}, A_{2}\right\}$. Since this point lies inside the fundamental domain for $\Gamma$, and is distinct from the fixed point $\left(p_{k}, q_{k}\right), k=0, \ldots, 3$, it is not conjugate to any of the fixed points of the elliptic elements of $G$, and does not project to a fixed point of the hyperelliptic involution.

\section{References}

[1] Chuckrow, V.: On Schottky groups with applications to kleinian groups. - Ann. of Math. 88, 1968, 47-61.

[2] Fenchel, W., and J. Nielsen: Discontinuous groups. - Unpublished.

[3] Ford, L.: Automorphic functions. - Chelsea, New York, 1951.

[4] JøRGensen, T.: On once punctured tori. - Preprint.

[5] Keen, L., H. E. Rauch, and A. T. Vasquez: Moduli of punctured tori and the accessory parameter of Lame's equation. - Trans. Amer. Math. Soc. 225, 1979, 201-229.

[6] KeEn, L.: Teichmuller spaces of punctured tori. - Preprint.

[7] Marden, A.: Schottky groups and circles. - Contributions to Analysis, Academic Press, New York-London, 1974, 273-278.

[8] MASkit, B.: A theorem on planar covering surfaces with applications to 3-manifolds. - Ann. of Math. 81, 1964, 341-355.

[9] Maskit, B.: A characterization of Schottky groups. - J. Analyse Math. 19, 1967, 227-230.

[10] Maskit, B.: On classification of kleinian groups II-signatures. - Acta Math. 138, 1977, 17-42.

Herbert H. Lehman College and

The Graduate School and University Center

of the City University of New York

Department of Mathematics

New York, New York 10468

USA

Received 15 June 1979

Revision received 2 November 1979 